\title{
Seconde partie : Prévention des maladies cardiovasculaires et apport en acides gras
}

Oléagineux, Corps Gras, Lipides. Volume 9, Numéro 4, 257-9, Juillet - Août 2002, Dossier : Equilibre entre acides gras insaturés : contributions à l'étude de la prévention des maladies cardio-vasculaires

\section{ARTICLE}

François Mendy. Passons au problème des apports en acides gras. Notre point de départ pour cette étude a été le suivant : l'effet des apports à des taux différents, en acide linoléique, en acide alphalinolénique, avait été étudié pendant des années ; restait le troisième acide gras : l'oléique. II était alors tentant de dire : fixons le taux de linoléique, fixons le taux d'alphalinolénique, et considérons l'acide oléique comme un acide gras neutre. Dans l'étude de Serge Renaud, le taux d'oléique était de $12,8 \%$ de l'apport calorique total. Puis survinrent les travaux de Rudel affirmant que l'oléate de cholestérol pouvait être très dangereux. Ceci était absolument prouvé au niveau cellulaire pour des raisons physico-chimiques et toute une série d'études chez le singe montraient que derrière l'oléate de cholestérol se jouait en fait l'augmentation des VLDL. Notre étude avait déjà commencé lorsque paru en 1997 un célèbre papier de Grundy soulignant l'absence de toute étude sur les taux d'acide oléique recommandables. II proposait 15 à $16 \%$ de l'apport calorique total en oléique et c'était justement les taux que nous étudiions. Le bien-fondé de notre objectif, d'analyser l'apparition d'éventuelles différences dans une population donnée, en faisant varier l'oléique avec des taux de linoléique et d'alphalinolénique fixés en fut ainsi confirmé.

Pierre Fossati. En France l'apport d'acides gras poly-insaturés est mal équilibré. Vous faites état de rapports $n-6 / n-3$ excessifs valant parfois jusqu'à 20 au lieu des valeurs habituellement recommandées tournant autour de 5. Si bien que j'ai une série de questions à vous poser à ce sujet. Que pensez-vous de la consommation très répandue d'huile de tournesol qui n'apporte pas d'alphalinolénique et simplement $20 \%$ d'acide oléique ? Ne pourrait-on pas commercialiser des mélanges d'huile mieux orientés dans la population ou consommer plus volontiers de l'huile de colza parfaitement équilibrée ou des associations d'huiles, olive et noix, olive et colza, olive et germes de blé ? C'est une question de santé publique importante : j'ai vu dans le Nord des gros mangeurs de beurre se transformer en gros mangeurs de margarines au-delà d'une dizaine de grammes, ceci en l'absence d'acide alphalinolénique.

Bernadette Delplanque. Les choses ont beaucoup évolué en quelques années. La part de consommation des mélanges d'huiles s'est fortement accrue, sous forme d'huile de table ou margarine aux dépens de l'huile de tournesol pure, bien que celle-ci soit encore une fraction importante de ces huiles de mélanges. Ces associations apportent des préparations plus équilibrées en acides gras, notamment grâce au colza. Et cette part croissante du colza amène ainsi de l'alphalinolénique douée d'une meilleure biodisponibilité que s'il s'agissait du soja. Les choses ont donc tendance à évoluer plutôt dans le bon sens ! 
François Mendy. À Lille, vous êtes au croisement du Nord et du Sud. Lorsque les enquêtes de consommation pour toute l'Europe ont été publiées, on a eu la grande surprise de constater que les Pays-Bas consommaient effectivement du linoléique dans une proportion représentant 8 à $9 \%$ de l'apport énergétique total (AET), et cela à cause de la margarine, alors qu'en France, la consommation de linoléique était de 3-4 \% de I'AET, ce qui n'est plus du tout élevé.

Nicole Combe. Cela se traduit par une consommation journalière de $8,3 \mathrm{~g}$ en moyenne au niveau national ${ }^{1}$, soit $4,1 \%$ de l'apport énergétique total, ce qui est une consommation correcte par rapport aux besoins. II a été bien montré que des situations de déficience en acides gras essentiels n- 6 sont corrigées par un apport minimum de 3-4 \% d'acide linoléique. En comparaison la consommation en Hollande et Belgique était en 1992, de $17 \mathrm{~g}$ à $19 \mathrm{~g}$ par jour ${ }^{2}$.

François Mendy. II est vrai toutefois que l'apport en alphalinolénique était sûrement insuffisant. J'ai même trouvé une population monastique pour laquelle le rapport $n-6 / n-3$ était de $60 / 1$. Mais en dehors de ces situations exceptionnelles et en dehors de la Hollande, on n'a jamais dépassé en France les $10 \mathrm{~g}$ de linoléique. Je ne voudrais pas que l'on tombe de Charybde en Scylla. II faut qu'il y ait un rapport de linoléique sur alphalinolénique de l'ordre de $6 / 1$ ou $5 / 1$ (les pédiatres le refusent pour différentes raisons et veulent rester à 12/1).

C'est un problème de mesure et d'équilibre. En mars 1982, Holman a montré que lorsqu'on donnait de l'alphalinolénique par voie intraveineuse chez un sujet carencé, à la dose de $54 \mathrm{mg} / \mathrm{kg}$ de poids, en deux ou trois mois tous les signes neurologiques disparaissent chez une petite fille qui avait été nourrie par alimentation parentérale uniquement avec de l'huile de carthame et qui avait constitué un syndrome neurologique.

"Malheureusement ", le mois suivant dans l'American Clinical Journal of Nutrition est paru l'article de Carlson sur les huiles de poisson et on a oublié le premier travail sur l'intérêt du précurseur : I'alphalinolénique. Dans ce premier travail Holman donne deux chiffres : $50 \mathrm{mg} / \mathrm{j}$ et un rapport de $6 / 1$ entre l'acide linoléique et l'alphalinolénique et ceci est systématiquement oublié. L'étude de Serge Renaud avait été faite avec un rapport de 6/1, ce que l'on avait vérifié chez Bernard Jacotot un peu avant. Ensuite il a été montré que si le linoléique est de $8 \%$ de l'apport calorique total, l'alphalinolénique ne se transformait qu'à $50 \%$. S'il est donné à $6 \%$ de l'apport calorique total, I'alphalinolénique est correctement transformé, ce qui semble être confirmé par l'étude d'Holman. II y a donc la nécessité d'un rapport de 6/1 entre les deux. Ce qui compte c'est en fait l'acide alphalinolénique biodisponible. Donné par voie intraveineuse il est " directement " biodisponible. Par voie orale, s'il est en position sn1 et sn3, Cloué et Bézard l'ont montré en 1989, il est brûlé préférentiellement. Dans le colza il y a $60 \%$ d'alphalinolénique en sn2 et dans le soja $33 \%$. Donc, si vous voulez obtenir $54 \mathrm{mg} / \mathrm{kg}$ de poids sous forme d'acide alphalinolénique biodisponible il faut en apporter $0,81 \%$ de l'apport calorique total d'une source colza, et 1,6 \% d'une source soja. Cette notion de biodisponibilité change énormément les choses.

Pierre Fossati. C'est un élément très important. II ne suffit pas d'apporter dans un aliment une certaine quantité d'alphalinolénique, ce qui compte c'est la quantité qui sera finalement biodisponible.

François Mendy. L'étude des sept pays a montré que le meilleur paramètre de protection était I'alphalinolénique dans les esters de cholestérol (chez les Crétois ils tournaient autour de $0,8 \%$ ). Un 
article de Simon a montré également que le facteur de prévention des accidents cérébraux vasculaires dans les populations étudiées était une consommation d'alphalinolénique dépassant les $0,5 \%$. D'où la proposition, de vérifier les pourcentages d'alphalinolénique dans les esters de cholestérol.

Pierre Fossati. II est bien évident que pouvoir explorer les esters de cholestérol en oléate, en linoléate ou linolénate est intéressant. Mais est-ce quelque chose de facilement réalisable ?

François Mendy. Cela permet juste de vérifier la validité des études épidémiologiques ! En fait l'alphalinolénique dans les esters de cholestérol est un critère de biodisponibilité.

Nicole Combe. Cette biodisponibilité de l'acide alphalinolénique doit être également pensée en termes de précurseur d'acides gras essentiels, puisque dans les esters de cholestérol les proportions d'acide eicosapentaénoïque (EPA) suivent celles de l'acide alphalinolénique.

Pierre Fossati. Cela permet de vérifier qu'il faut absorber un acide alphalinolénique biodisponible en bonne quantité. Peut-on envisager la possibilité de risques en cas d'excès de consommation, comme a pu être établi le parfait parallélisme entre consommation de linoléique, composition de tissus adipeux en linoléique et risques de coronaropathies ?

Nicole Combe. On a vu qu'il était possible, selon le régime d'augmenter le pourcentage d'acide alphalinolénique dans les esters de cholestérol $(\mathrm{EC})$ jusqu'à environ $3 \%$ des acides gras totaux.

François Mendy. Pour Mensik et Katan, le linoléique dans les esters de cholestérol est très nettement corrélé à l'apport alimentaire en linoléique. Cela dit chez les nourrissons prématurés au lait maternel il n'y a pas de corrélation entre le linoléique présent dans le lait et les esters de cholestérol plasmatiques, alors qu'il y a une corrélation entre l'alphalinolénique du lait et les esters de cholestérol. II y a plusieurs niveaux d'équilibre : un équilibre oléique/linoléique qui module le pourcentage de linoléique ou d'oléique dans les esters de cholestérol, et un troisième paramètre qui est l'alphalinolénique qui est, de loin, celui qui rentre le plus facilement dans les esters de cholestérol et qui pourrait avoir un effet sur le pourcentage de linoléique présent dans ces esters (c'est ce que nous avons publié avec Bernard Descomps dans le travail chez les prématurés).

En fait il y a une controverse : Rudel dit que l'oléate de cholestérol est dangereux, tous les spécialistes des problèmes d'oxydation disent que c'est le linoléate de cholestérol qui est responsable de la toxicité des LDL oxydées, et l'acide alphalinolénique, dont on n'a pas parlé pendant longtemps, mais dont on commence à mesurer l'importance dans la régulation de ce système.

Au final, deux points importants : I'alphalinolénique en Sn2 va dans les esters de cholestérols même en quantité relativement faible, et la composition des esters de cholestérol est un bon marqueur (on n'a pas besoin de biopsie).

Nicole Combe. On y observe bien la relation existant entre acide alphalinolénique et EPA.

Pierre Fossati. Quand l'EPA augmente, c'est que l'alphalinolénique a été transformé. S'il y a trop de linoléique tout est bloqué. Apparaît bien cette nécessité de l'équilibre entre le linoléique et I'alphalinolénique. Est-on sûr d'un risque de consommation excessif d'acide oléique ? Vous avez rapporté chez le singe et les hamsters, les perturbations des LDL et du cholestérol. Quelle était alors 
la valeur du rapport $n-6 / n-3$ ? Sur le plan épidémiologique des risques corrélés à une forte consommation d'huile d'olive n'ont jamais été rapportés. En Grèce la consommation moyenne est de $60 \mathrm{~g} / \mathrm{j}$, soit un apport de $40 \mathrm{~g}$ d'acide oléique et $7 \mathrm{~g}$ d'acide linoléique représentant des conditions d'assez bon équilibre. Une alimentation variée avec moins de graisses saturées et plus de poisson, moins de viande, du pourpier, des escargots, des amandes et des noix et on a tout l'acide alphalinolénique nécessaire.

François Mendy. Vous avez fait le calcul et vous tombez sur $40 \mathrm{~g}$ par jour. Or la dernière édition des apports nutritionnels conseillés (ANC) préconisent $50 \mathrm{~g}$ et quand vous regardez cette enquête sur la Grèce, vous vous apercevez que ce sont les Grecs, riches, qui consomment $40 \mathrm{~g}$ et plus d'acide oléique.

Bernadette Delplanque. La diète méditerranéenne " light " comme on l'appelle parfois maintenant, correspond au régime traditionnel des pauvres d'autrefois. Dans l'étude de Lyon de Serge Renaud la consommation était calquée sur le régime crétois avec seulement $28 \mathrm{~g}$ d'oléique, pour 2000 calories représentant $12,9 \%$ de l'apport énergétique total et $8 \mathrm{~g}$ de linoléique. Dans notre étude, nous nous sommes placés de $28 \mathrm{~g}$ à un peu plus de $40 \mathrm{~g}$ d'acide oléique.

Pierre Fossati. Finalement vous êtes restés dans la fourchette. D'où la question : quelle erreur diététique faut-il faire pour rencontrer un excès d'acide oléique pathogène ?

François Mendy. Il faut suivre... les ANC ce qui implique de faire un gros effort... En fait, les études de Rudel montrent que la situation est dangereuse autour de $24 \%$ de l'apport énergétique total. Des études chez l'homme, une étude européenne (Carmena), a été faite à $22 \%$ d'oléique versus linoléique (tournesol). Et à la surprise générale les plus mauvais résultats sont obtenus avec l'huile d'olive à $22 \%$. En fait là il pose le problème du lien avec les protéoglycanes de la paroi artérielle. De même une étude belge montre que lorsque le pourcentage d'oléate de cholestérol augmente, la fluidité change considérablement dans certaines cellules. II y a donc une zone de flexibilité comprise entre les $28 \mathrm{~g}$ de Serge Renaud et un peu plus de vos $40 \mathrm{~g}$.

Pierre Fossati. II faut être pondéré en nutrition également vis-à-vis des acides gras. Dans l'étude numéro 3 que nous allons faire dans le cadre du programme de Fleurbaix Laventie, nous allons beaucoup insister sur l'introduction des acides gras $n-3$, dans l'esprit de ce que j'ai écrit il y a quelques années. Pour revenir à l'acide linoléique, c'est un acide gras essentiel qui doit être apporté entre 5 et $10 \mathrm{~g}$.

Bernadette Delplanque. Nos études précédentes ont montré qu'un minimum de 7 à $13 \mathrm{~g}$ était nécessaire pour préserver des valeurs basses de LDL-C.

Pierre Fossati. Je crois que tout dépend de la façon dont on raisonne. Je me souviens d'une étude qui montrait qu'il n'y avait pas plus d'oxydation avec l'huile de tournesol qu'avec l'huile d'olive c'était une étude espagnole à condition d'apport suffisant en anti-oxydants, en vitamine E par exemple. II est bien évident que si on a un peu plus d'acide linoléique mais que l'on a suffisamment d'alphalinolénique il n'y a pas de problème.

Bernadette Delplanque. C'était exactement le sens de ma réflexion précédente : il ne faut pas diminuer le linoléique mais plutôt augmenter la consommation d'alphalinolénique. Dans ces conditions il n'y a pas de problème de compétition entre les 2 voies n- 6 et n-3 pour la transformation 
en dérivés supérieurs, si importants dans la prévention des problèmes d'inflammation et/ou d'agrégation. II n'y a pas de problème non plus si le risque d'oxydation est limité, ce qui veut dire que I'on épure rapidement les particules riches en triglycérides, ce qui implique des valeurs d'apo-CIII normales, non limitantes sur les processus d'épuration des particules qui les transportent. Pour le maintien d'un métabolisme normal, la fourchette des mécanismes de régulation est quand même relativement large, tout comme la gamme d'apports en acides gras, à condition de n'en privilégier et de n'en oublier aucun parmi ceux que nous avons cités. Mais à partir du moment où (" génétiquement ") on a une épuration difficile ou pas assez de vitamine $E$ tout devient compliqué et on risque de basculer dans une pathologie ou une autre qui sera fonction du contexte physiologique de chaque individu. C'est certainement là qu'apparaissent tout l'intérêt et le rôle important des micro-nutriments, comme le souligne justement Bernard Jacotot, à cette nuance près, qu'à mon avis, la qualité des acides gras demeure capitale, au vu de leurs multiples fonctions sur le métabolisme des lipides et sur le plan moléculaire.

François Mendy. Lorsque les apports se font sous forme équilibrée, les résultats sont meilleurs. C'était un peu l'objectif de cette étude. Le dernier point était celui-ci : pourquoi parmi ces moines normaux, certains ont déjà des cinétiques d'exception comme s'ils étaient déjà à l'extrême limite des possibilités de régulation?

Pierre Fossati. L'hérédité ?

François Mendy. Certains sujets ont de grandes capacités de régulation, mais pas tous. Il faut donc faire très attention lorsqu'on lance des recommandations d'ordre général. Il y a à peu près $20 \%$ de la population qui n'a pas une aussi grande souplesse de régulation que les autres. Et ceux-là feront probablement des accidents vasculaires ou... des syndromes plurimétaboliques... Votre étude Fleurbaix Laventie devrait vous permettre de détecter ces sujets potentiellement à risque, et secondairement évaluer les facteurs de risque ou d'environnement qui les ont fait basculer.

Pierre Fossati. Il y a des gens qui « virent » et il faudrait pouvoir dépister ceux qui vont « virer ».

François Mendy. La vraie question est celle-là.

Pierre Fossati. Dans cette étude, nous disposons de l'hérédité (accidents vasculaires du père ou de la mère...) et ceux qui régulent mal, s'ils ne mangent pas parfaitement équilibrés, seront susceptibles eux aussi de faire des accidents parce que des gens qui mangent déséquilibré et qui vont bien, il y en a tout de même beaucoup.

Bernadette Delplanque. ... jusqu'à un certain âge.

Pierre Fossati. Ça va bien jusqu'au moment où ça va mal !

Propos recueillis par Jean-Claude Icart avec la collaboration très précieuse de Madame Bernadette Delplanque 
Notes:

${ }^{1}$ Étude TRANSFAIR, 1993-94.

2 Étude TRANSFAIR. 